\title{
O Programa de Pós-Graduação em Psicologia da Universidade Federal do Amazonas: proposta e perspectivas para a região Amazônica
}

Gisele Cristina Resende. Universidade Federal do Amazonas lolete Ribeiro da Silva. Universidade Federal do Amazonas Marcelo Gustavo Aguilar Calegare. Universidade Federal do Amazonas

\section{Resumo}

O presente artigo objetiva apresentar e discutir o Programa de Pós-Graduação em Psicologia da Universidade Federal do Amazonas (PPGPSI-UFAM) em sua proposta formativa e perspectivas para a região amazônica diante do cenário da pós-graduação nacional. Apresentamos também suas origens e características atuais, o perfil esperado e pesquisa com egressos dos 10 anos de PPGPSI, as parcerias nacionais e internacionais, as principais produções de 2017-2018 e a inserção social. Revelamos a dificuldade de elevar as produções científicas e realizar preenchimento adequado do Portal Sucupira. E indicamos alguns desafios a serem superados: isolamento e desconhecimento da Amazônia pelo restante do país, falta de incentivos financeiros para a região norte, desconsideração das especificidades amazônicas para financiamentos, adoção de novas epistemologias conforme olhar regional, fortalecimento do PPGPSI por parcerias nacionais e internacionais.

Palavras-chaves: pós-graduação; psicologia; Amazônia.

\begin{abstract}
The Postgraduate Program in Psychology of the Federal University of Amazonas: Proposal and Perspectives for the Amazon Region. This article aims to present and discuss the Program the Postgraduate Program in Psychology of the Federal University of Amazonas (PPGPSI-UFAM) in its formative proposal and perspectives for the Amazon region facing the national postgraduate scenario. We also present its origins and current characteristics, the expected profile and research with graduates of the 10 years of PPGPSI, the national and international partnerships, the main productions of 2017-2018 and the social insertion. We reveal the difficulty of raising the scientific productions and realizing adequate fulfillment of the Sucupira Portal. And we indicate some challenges to be overcome: isolation and ignorance of the Amazon for the rest of the country, lack of financial incentives for the Northern region, disregard of the Amazon specificities for financing, adoption of new epistemologies according to regional perspective, strengthening of PPGPSI by national and international partnerships.
\end{abstract}

Keywords: postgraduated training; psychology; Amazon region.

\section{Resumen}

El Programa de Postgrado en Psicología de la Universidad Federal de Amazonas: Propuesta y Perspectivas para la Región Amazónica. El presente artículo tiene por objeto presentar y discutir el Programa. el Programa de Postgrado en Psicología de la Universidad Federal del Amazonas (PPGPSI-UFAM) en su propuesta formativa y perspectivas para la región amazónica ante el escenario nacional del postgrado. Presentamos también sus orígenes y características actuales, el perfil esperado e investigación con egresados de los 10 años de PPGPSI, las alianzas nacionales e internacionales, las principales producciones de 2017-2018 y la inserción social. Revelamos la dificultad de elevar las producciones científicas y realizar el llenado adecuado del Portal Sucupira. E indicamos algunos retos para superación: el aislamiento y desconocimiento de Amazonia por el resto del país, la falta de incentivos económicos para la región norte, la desconsideración de las especificidades amazónicas para financiamientos, la adopción de nuevas epistemologías conforme mirada regional, el fortalecimiento del PPGPSI por alianzas nacionales e internacionales.

Palabras clave: posgrado; psicología; Amazonia. 


\section{A Pós-Graduação no Brasil e na Região Norte}

Em 2019, o Programa de Pós-Graduação em Psicologia (PPGPSI) na Universidade Federal do Amazonas (UFAM) completou 10 anos de existência, havendo pontos fortes e muitos desafios a serem superados para chegarmos a uma melhor excelência. Neste artigo de caráter descritivo e analítico, temos por objetivo apresentar e discutir o PPGPSI em suas origens, proposta formativa, características atuais, perspectivas e desafios para a região amazônica diante do cenário da pós-graduação nacional.

Para alcançar nosso propósito, realizamos breve revisão crítica de literatura referente ao debate da pós-graduação no Brasil e região Norte, utilizando também pesquisa documental em documentos importantes para tratar de nosso PPGPSI: plano nacional de pós-graduação 2011-2020 (Ministério da Educação, 2010); documento de área - Psicologia (Ministério da Educação, 2016); proposta pedagógica do programa (Universidade Federal do Amazonas [Ufam], 2008); regimento interno do PPGPSI (Ufam, 2016); relatórios enviados na plataforma sucupira em 2017 (Coordenação de Aperfeiçoamento de Pessoal de Nível Superior [CAPES], 2017) e 2018 (CAPES 2018); relatório da avaliação dos 10 anos do PPGPSI com pesquisa de egressos (Ufam, 2019). Tal pesquisa teve como objetivo conhecer o perfil e as áreas de atuação profissional dos mestres formados no PPGPSI, indicando a contribuição que o programa oferece à sociedade ao formar metres na área da Psicologia.

Tecidas essas considerações introdutórias, propomos o seguinte percurso neste artigo: contextualizar a pós-graduação no Brasil e na região Norte, enfocando a área da Psicologia; apresentar e discutir a trajetória e características do PPGPSI, o perfil esperado e dos egressos do PPGPSI na pesquisa dos 10 anos, as parcerias intra e interinstitucionais, as principais produções e inserção social. Finalizamos com análises das necessidades e perspectivas futuras enquanto um PPG do Norte, indicando caminhos para sua manutenção e fortalecimento.

A pós-graduação no Brasil começou a existir a partir da década de 1930, com professores europeus em missões acadêmicas proporcionadas pelos governos europeus ou para fugir dos impactos da II Guerra Mundial na Europa. Naquele momento, no Brasil não havia uma política institucionalizada para a pós-graduação e os professores faziam parcerias com as universidades e criavam grupos de estudo e pesquisas, as cátedras, dando-se assim o início da pós-graduação no Brasil. Em 1951, foi criada a Coordenação de Aperfeiçoamento de Pessoal de Nível Superior (CAPES), fundação do Ministério da Educação (MEC), para expandir e consolidar a pós-graduação stricto sensu. Em 1965, o governo brasileiro, por meio do Ministério da Educação, criou um plano para a pós-graduação, pois o país precisava qualificar seus professores universitários e era uma alternativa de nacionalizar este nível de ensino, assim pensou-se em programas de mestrados e doutorados. Em 1968, com a reforma do ensino superior, adotou-se um modelo norte-americano para as universidades brasileiras e a pós-graduação foi institucionalizada e relacionada aos departamentos das universidades (Balbachevsky, 2005).

Após a institucionalização da pós-graduação, políticas de apoio e incentivo vêm sendo criadas para a consolidação deste nível de ensino por meio dos Planos Nacionais da Pós-Graduação (PNPG), documentos que norteiam as políticas desta área. O Brasil teve cinco PNPGs: 1975-1979, 1982-1985, 1986-1989, 2005-2010 e 2011-2020. Em linhas gerais, pode-se dizer que inicialmente o foco foi a formação de professores ao magistério superior e pesquisadores/cientistas para atender às demandas da sociedade, passando pela integração das universidades com o setor produtivo, expansão e melhoria dos sistemas de pós-graduação, chegando-se ultimamente à internacionalização, valorização da multi/interdisciplinaridade e integração com todos os níveis de ensino (Ministério da Educação, 2010).

Como apontado por Freitas e Souza (2018) a pós-graduação stricto sensu no Brasil é a grande responsável pela produção de conhecimento científico nos últimos 45 anos, favorecendo o desenvolvimento do país. Calegare e Tamboril (2017), corroborando com isso, esclarecem que tal produção acontece prioritariamente nos programas de pós-graduação (PPGs), que se encontram majoritariamente nas instituições de ensino superior públicas, especialmente na rede federal. E explicaram que, mais especificamente na região Norte, com exceção dos estados do Pará e Amazonas, houve um tardio processo de instalação de universidades federais: Universidade Federal Rural da Amazônia (UFRA), Pará, em 1945; Universidade Federal do Pará (UFPA), em 1957; Universidade Federal do Amazonas (UFAM), em 1962 (apesar de ter sido fundada em 1909 com outro nome); Universidade Federal do Acre (UFAC),

Estudos de Psicologia, 24(2), abril a junho de 2019, 125-135 
em 1971; Universidade Federal de Rondônia (UNIR), em 1982; Universidade Federal de Roraima (UFRR), em 1985; Universidade Federal do Amapá (UNIFAP), em 1990; Universidade Federal de Tocantins (UFT), em 2000; Universidade Federal do Oeste do Pará (UFOPA), em 2009. Com isso, houve uma consequente demora na criação de PPGs na região, o que teve como resultados: a demora na formação de recursos humanos locais; escassez de investimentos atrativos para fixação de mão de obra qualificada; expansão de cursos de graduação e pós-graduação latu sensu pela iniciativa privada, com cursos de curta duração.

$\mathrm{Na}$ área da Psicologia, pertencente ao colégio de Humanidades na classificação da CAPES, o primeiro PPG foi o da Pontifícia Universidade Católica em São Paulo (PUC-São Paulo), criado no ano de 1966. Em 1970, foi criado na Universidade de São Paulo (USP) o programa nas áreas de Psicologia Experimental e Clínica. Na década de 1980, foram criados programas na região nordeste (Pernambuco, Paraíba e Rio Grande do Norte), no sul (Rio Grande do Sul) e na região norte (Pará), o que expande a pós-graduação, mas que ainda assim deixava de atender todas as necessidades nacionais, considerando o Brasil rico em extensão territorial e diversidades regionais. Nos anos 1990 e 2000 há expansão em outros estados dessas regiões, assim como em novas regiões.

Novamente abordando a região Norte, Calegare e Tamboril (2017) mostram que o primeiro PPG criado, em 1987 foi o mestrado em Teoria e Pesquisa do Comportamento (Psicologia), na UFPA, com doutorado aberto em 1999 - nota 5 atualmente. Em 2005, na mesma universidade, veio o mestrado do PPG em Psicologia, seguido do doutorado em 2014 - nota 4 atualmente. Em 2009, foram criados PPGs em Psicologia tanto na UFAM quanto na UNIR (Mestrado em Psicologia - MAPSI), que até o presente possuem apenas mestrado e são nota 3. Finalmente, em 2014 foi criado o PPG em Neurociência e Comportamento, também na UFPA, na área básica de Psicobiologia, com apenas mestrado e nota 3.

De acordo com o Documento de Área da Psicologia (Ministério da Educação, 2016), a área possuía à época de sua publicação 53 doutorados, 79 mestrados acadêmicos e sete mestrados profissionais, totalizando 139 cursos de pós-graduação stricto sensu. Estes cursos encontram-se no Brasil com os seguintes quantitativos em ordem decrescente: Sudeste (40 PPGs, com 69 cursos de mestrado e doutorado, ou $46,5 \%$ do total; sendo que no estado de São Paulo existem 20 programas, representando $23,3 \%$ do total da área); Nordeste (18 PPGs e 27 cursos, 20,9\% do total; Sul (12 PPGs e 18 cursos, $14 \%$ do total; dos quais sete estão no estado do Rio Grande do Sul); Centro-Oeste (11 PPGs e 18 cursos, $12,8 \%$ do total; dos quais seis deles encontram-se no Distrito Federal); Norte (cinco PPGs e sete cursos, 5,8\% do total).

Nota-se que não há PPGs em Psicologia nos estados do Acre, Amapá, Roraima e Tocantins. Diante desse cenário de assimetria intrarregional e disparidade inter-regional, Calegare e Tamboril (2017) demonstram que, apesar da proporção de títulos de mestrado e doutorado concedidos no Brasil ter crescido significativamente desde os anos 1990, dificilmente a região Norte alcançará as condições necessárias para superação dessas desigualdades tendo em vista que, mesmo tendo sido apontada como prioridade em documentos oficiais dos governos, por meio de planos e programas da política nacional de educação, os resultados dessas ações ainda são incipientes.

A partir deste cenário visualiza-se a necessidade de fortalecimento e ampliação dos PPGs em Psicologia na região Norte, para que haja a construção de conhecimentos científicos que atendam às demandas regionais. Essa é uma das missões assumidas pelo PPGPSI-UFAM, como será apresentado a seguir.

\section{Trajetória e características do PPGPSI-UFAM}

O PPGPSI surgiu a partir do reconhecimento, por professores da Faculdade de Psicologia (FAPSI) e dos egressos do curso de graduação em Psicologia, de que havia uma demanda estadual por cursos de pós-graduação stricto sensu e por ampliação da formação de recursos humanos e da produção acadêmica de alto nível em Psicologia, tendo como foco a realidade amazonense. A implantação do PPGPSI começou a ganhar contorno com a realização do doutoramento por parte de docentes da FAPSI, nos anos 2000, seja de maneira independente ou pelo doutorado interinstitucional entre a UFAM e a Universidade de São Paulo/Ribeirão Preto DINTER UFAM/USP-RP. Graças ao esforço desses/as professores/as, foi possível reunir condições suficientes para solicitar a abertura do curso de pós-graduação, que foi aprovado pela CAPES em outubro de 2008. Assim, o PPGPSI foi criado em 2009 por meio da publicação do Parecer $n^{\circ}$ 122/2009 do MEC, publicado no D.O.U. 
de 19/6/2009, Seção 1, pág. 23 a 28. Até o presente, continua sendo o único curso de mestrado em Psicologia do Amazonas e, no primeiro semestre de 2019 , contava com 14 docentes: 12 permanentes e dois colaboradores (Ufam, 2008).

Nosso quadro docente se assemelha a outros da região norte: uma parte do quadro é composta por professores que fizeram Minter e/ou Dinter, com longa experiência na universidade, porém já desacelerando na carreira docente e com vistas à aposentadoria; outra parte que está chegando recentemente à universidade, já com doutorado e em geral recém-doutores, e que possuem pouca experiência e produção acadêmica.

O PPGPSI assumiu a responsabilidade de pensar as problemáticas da região amazônica, tendo em vista a produção e expansão do conhecimento científico, por meio da formação de quadros profissionais qualificados. Nesta perspectiva, o PPGPSI vem buscando responder às demandas e políticas científicas locais e nacionais, efetivamente formando pesquisadores e docentes para o ensino superior (há 10 cursos de graduação em Psicologia em Manaus, sendo nove pertencentes à rede privada de ensino superior), bem como qualificando psicólogos para atuarem nas instituições públicas e privadas do Amazonas (Sistema Único de Saúde, Sistema Educacional, Sistema Único de Assistência Social, Sistema de Justiça, no Polo Industrial e Tecnológico, dentre outros). A formação ético-crítica dos discentes vem se refletindo em uma produção local que articula os conhecimentos teóricos e metodológicos afeitos ao conhecimento universal da Psicologia, com o estudo de temáticas vinculadas à realidade social regional. Por outro lado, o PPGPSI já desponta como parte importante do compromisso social de produção autóctone de conhecimento na região, socialmente referenciada e sensível às especificidades do contexto e população amazônica (CAPES, 2017, 2018).

Temos como objetivos de nosso PPGPSI: (a) produzir conhecimentos na área da Psicologia, voltados para a compreensão e intervenção nas questões ligadas à realidade amazônica, no nível de mestrado; (b) formar pessoas qualificadas nos referenciais teórico-metodológicos da Psicologia para o exercício no magistério em ensino superior, na pesquisa científica, na extensão e na inovação tecnológica e social; (c) capacitar profissionais para atuar em instituições públicas ou privadas, capazes de utilizar conhecimentos teórico-técnicos inovadores que influenciem nas políticas públicas e na busca do bem-estar e para atuarem como consultores e assessores qualificados para questões relativas à gestão e políticas sociais e de saúde, direitos humanos e justiça social (CAPES, 2018; Ufam, 2016, 2019).

Considerando a amplitude das demandas identificadas, optou-se por uma proposta de programa que incluísse todos os docentes aptos ao credenciamento e considerando a diversidade de áreas de formação foram delineadas duas linhas de pesquisa abrangentes. Essa decisão da adoção de linhas de pesquisa abrangentes decorreu, essencialmente, do pequeno número de professores/as credenciados do Programa nos seus primeiros 10 anos de existência e da diversidade de áreas de formação dos/as mesmos/as. Em 2013, o PPGPSI realizou uma autoavaliação e, contando com o apoio de pesquisadores de outras regiões, reformulou as linhas de pesquisa de forma a contemplar mais explicitamente o contexto amazônico nas suas descrições e, consequentemente, adotar uma identidade regional mais definida. Avaliou-se que esse foi um momento importante para o fortalecimento da identidade do PPGPSI, destacando-se que as contribuições de pesquisadores de outras regiões demonstravam a importância da solidariedade de PPGs mais experientes. O redesenho das linhas de pesquisa também representou em um sinal de maturidade do grupo que passou a assumir com mais segurança sua identidade amazônica. Atualmente as linhas de pesquisa são descritas da seguinte maneira:

- Linha 1 - Processos Psicossociais: trata de pesquisas relacionadas à produção da subjetividade presente no comportamento humano nas diversas dimensões psicossociais, na relação entre pares ou entre grupos sociais, ou na relação pessoa-ambiente, buscando compreender essa produção psicossocial e cultural numa perspectiva histórica e socioambientalmente contextualizada na realidade amazônica.

- Linha 2 - Processos Psicológicos e Saúde: integra pesquisas voltadas para a promoção de saúde e práticas terapêuticas em diferentes espaços (políticas públicas de saúde, promoção de saúde no trabalho, na educação), partindo de diversas matrizes teóricas e metodológicas, voltadas para a produção de conhecimento sensível às especificidades amazônicas e implicado na transformação social.

Desde a criação do PPGPSI, os laboratórios de pesquisa foram pensados como espaços aglutinadores das ações formativas. Ao longo do tempo, com a ampliação do número de docentes credenciados também 
se ampliou o número de laboratórios. Atualmente existem oito laboratórios aglutinados em torno das linhas de pesquisa. Estão ligados à linha 1: Laboratório de Desenvolvimento Humano e Educação (LADHU); Laboratório de Intervenção Social e Desenvolvimento Comunitário (LABINS); Laboratório de Avaliação Psicopedagógica, Educacional, Histórico Cultural da Amazônia (LAPESAM - UFAM campus de Humaitá). À linha 2 estão vinculados: Laboratório de Psicologia da Saúde (LAPSA); Laboratório de Psicologia FenomenológicoExistencial (LABFEN); Laboratório de Psicodinâmica do Trabalho (LAPSIC); Laboratório de Avaliação Psicológica (LAP); Laboratório de Intervenção em Ciências Cognitivas (LABICC) (CAPES, 2018, 2017; Ufam, 2008, 2016, 2019).

\section{Perfil dos egressos}

Pretende-se que os egressos do PPGPSI saiam instrumentalizados com uma visão teórica, ética e crítica a respeito da realidade regional amazônica, seja nos aspectos da vida urbana de Manaus ou cidades do interior, nos aspectos relacionados às comunidades rurais e distintos grupos étnico-raciais, ou das vicissitudes regionais que se expressam na vida das pessoas nesse trânsito entre cidade e floresta. A partir dessa visão que integra a história pessoal, o contexto histórico-cultural regional e o espaço amazônico, espera-se que os egressos tenham competências profissionais que os habilitem a atuar em instituições públicas e privadas, em políticas públicas ou no ensino superior de modo a gerar transformações para uma sociedade melhor. Pretende-se ainda que saibam elaborar e conduzir projetos de pesquisa e intervenção, de maneira a realizar mediação entre saber científico e demandas sociais, com um olhar sobre o contexto e necessidades amazônicas. Assim, nosso foco é habilitar profissionais capacitados a trabalhar pela melhoria da sociedade regional, especialmente nas áreas da saúde, direitos humanos e justiça social (CAPES, 2017, 2018; Ufam, 2019).

Nestes 10 anos de PPGPSI, houve 115 defesas de mestrado. Será que esses mestres em Psicologia estão cumprindo com nossa proposta? Para responder a essa pergunta, realizamos uma pesquisa com os egressos, para conhecer os caminhos percorridos por eles e verificar se o perfil que delineamos e os objetivos do PPGPSI têm sido alcançados. Para tanto, no final de 2018 foi elaborado um formulário eletrônico no GoogleDocs, contendo as seguintesquestões: identificação, ano da turma de ingresso, ano de conclusão do mestrado, tipo de vínculo empregatício atual (CLT, Servidor Público na esfera Federal, Estadual, Municipal,
Aposentado, Colaborador, Bolsista, Outro, Sem vínculo); setor de atuação (ensino e pesquisa, políticas públicas saúde, educação, assistência social, jurídica, outro); área de atuação (saúde, educação, assistência social, jurídica, organizacional, trabalho, clínica, comunitária, outro); informação a acrescentar (registro de patente, artigos científicos, experiência inovadora). O formulário foi enviado aos 115 egressos até aquele momento, das oito turmas que concluíram o curso, via e-mail cadastrado no PPGPSI, entre os meses de setembro e outubro de 2018. Os dados foram organizados em planilha, utilizando o programa Excel, e analisados por meio de estatística descritiva e categorização das respostas à pergunta aberta (Bardin, 2011).

Retornaram 69 formulários respondidos pelos egressos, representando $60 \%$ de respondentes, resultado considerado satisfatório tendo em vista que após nove anos de curso os mestres foram contatados para fornecer informações sobre a contribuição do mestrado para a sua vida profissional. A tabela 1 demonstra a turma e o ano de conclusão dos egressos participantes da pesquisa.

Tabela 1. Alunos Respondentes por Ano de Entrada e Conclusão

\begin{tabular}{cccc}
\hline Entrada & Conclusão & \multicolumn{2}{c}{ Alunos Respondentes } \\
\hline & & Freq. $\left(\mathbf{n}^{\circ}\right)$ & Percentual (\%) \\
\hline 2009 & 2011 & 7 & 10,1 \\
2010 & 2012 & 5 & 7,2 \\
2011 & 2013 & 11 & 15,9 \\
2012 & 2014 & 9 & 13,0 \\
2013 & 2015 & 9 & 13,0 \\
2014 & 2016 & 10 & 14,5 \\
2015 & 2017 & 7 & 10,1 \\
2016 & 2018 & 11 & 15,9 \\
\hline Total & & 69 & 100 \\
\hline
\end{tabular}

Observa-se que os egressos da $1^{\text {a }}$ turma de mestrado iniciado em 2009 representam 10,1\% dos respondentes, apontando que mesmo após vários anos de conclusão do curso, os primeiros mestres formados mantêm o vínculo com o PPGPSI-UFAM, pois responderam o formulário. Ainda, 15,9\% dos respondentes concluíram o curso de mestrado no ano 2018 ( $8^{\mathrm{a}}$ turma), mantendo o vínculo por meio de contato eletrônico e participação em laboratórios de pesquisas. Neste aspecto, os laboratórios desenvolvem meios próprios de manutenção do vínculo e registro das atividades realizadas pelos egressos, as quais são informadas à secretaria e coordenação do PPGPSI por ocasião do 
relatório anual a ser entregue à CAPES. Por meio dessa vinculação, a participação dos egressos nas atividades dos laboratórios tem sido incentivada, buscando-se envolvê-los em publicações científicas.

O formulário também averiguou o vínculo empregatício do egresso, pois pretendia-se saber se o egresso do PPGPSI está atuando na área da Psicologia e como o mestrado contribuiu para a inserção no mercado de trabalho, conforme demonstrado na Tabela 2.

Tabela 2. Vínculo Trabalhista do Egresso

\begin{tabular}{lcc}
\hline \multicolumn{1}{c}{ Vínculo } & Freq. $\left(\mathrm{n}^{\circ}\right)$ & Percentual $(\%)$ \\
\hline Servidor Federal & 9 & 13,0 \\
Servidor Estadual & 17 & 24,6 \\
Servidor Municipal & 6 & 8,7 \\
Servidor Estadual e Municipal & 9 & 13,0 \\
Autônomo & 1 & 1,4 \\
CLT & 15 & 21,7 \\
Sem vínculo & 9 & 13,0 \\
Bolsista & 3 & 4,3 \\
\hline Total & 69 & 100,0 \\
\hline
\end{tabular}

Os egressos em sua maioria são servidores públicos nas esferas federal, estadual e municipal, totalizando 59,3\% dos respondentes. Além disso, os mestres formados pelo PPGPSI atuam principalmente em setores ensino e pesquisa $(47,8 \%)$ e nas políticas públicas (saúde, educação, assistência social e jurídica) (31,9\%), restando apenas uma parcela que atua em outras áreas (Psicologia Clínica, comunitária e do trabalho) (20,3\%).

Os dados apontam que o PPGPSI cumpre os objetivos de formar recursos humanos na Amazônia, com a competência de analisar a conjuntura regional e nacional, capaz de planejar e elaborar ações para a articulação entre conhecimento científico em Psicologia e as demandas de intervenção. Alguns(as) egressos(as) tornam-se referência profissional na cidade e no estado do Amazonas nas diversas áreas de atuação profissional, como relata uma egressa:

O mestrado abriu portas para a vida acadêmica, dei aula por seis anos em graduação do curso de Psicologia em instituições privadas e como professora substituta na UFAM. Hoje dou aula em curso de pós-graduação, oriento estagiários, além de ser estatutária como psicóloga em cargos públicos. Referência na área, sempre chamada para diversos eventos científicos em Psicologia e Saúde. (Ufam, 2019, p. 7)
O mestrado também possibilitou a alguns egressos o ingresso em PPGs com doutorado fora do estado do Amazonas, em PPGs na USP, Universidade Federal do Rio Grande do Norte (UFRN), Universidade Federal de Santa Catarina (UFSC), Universidade Federal de Minas Gerais (UFMG). Os que permaneceram no Amazonas optaram por cursar doutorados em PPGs da UFAM, como aqueles em: Antropologia; Educação; Sociedade e Cultura. Vejamos um relato de um egresso, a respeito da continuidade na carreira de pesquisa: "...O mestrado no Programa de Pós-Graduação em Psicologia na Universidade Federal do Amazonas me proporcionou o ingresso no doutorado na Universidade de São Paulo, pois contribuiu para minha formação em pesquisa e contribuiu para a elaboração de meu currículo". (Ufam, 2019, p. 7)

Em suma, a pesquisa com egressos demonstrou que o PPGPSI tem cumprido seus objetivos de: produzir conhecimentos na área da Psicologia voltados para a compreensão e intervenção nas questões ligadas à realidade amazônica; formar recursos humanos qualificados teórico-metodologicamente na área da Psicologia para o exercício no magistério em ensino superior, na pesquisa científica, na extensão e na inovação tecnológica e social; capacitado profissionais para atuar em instituições públicas ou privadas, influenciando na elaboração de políticas públicas para o bem-estar social e desenvolvimento regional (Ufam, 2016, 2019).

\section{Parcerias intra e interinstitucionais}

Temos buscado estabelecer parcerias dentro da própria UFAM, com instituições em Manaus, na região Norte, em outras regiões do Brasil e até mesmo no nível internacional. No âmbito intrainstitucional, alguns docentes têm participado em atividades dos laboratórios, núcleos de pesquisa, bancas e projetos em conjunto com docentes de outros PPGs da UFAM, seja por aqueles com recursos financeiros patrocinados por agências financiadoras ou por aqueles autofinanciados. Entre esses PPGs, destacamos: Antropologia Social, Ciências Ambientais e Sustentabilidade na Amazônia, Cirurgia, Comunicação, Educação e Serviço Social.

No âmbito interinstitucional na cidade de Manaus, temos incentivados os alunos a cursarem disciplinas em outros PPGs da UFAM ou nas demais instituições que oferecem pós-graduação em Manaus: a Universidade Estadual do Amazonas (UEA), Instituto Nacional de Pesquisas da Amazônia (INPA) ou no Instituto Leônidas 
e Maria Deane - Fundação Oswaldo Cruz (ILMD-Fiocruz Amazônia). Em muitos casos, a indicação é feita por sugestão do orientador que conhece alguém ou o PPG dessas outras instituições de Manaus e que mantém alguma vinculação com os mesmos.

A respeito das parcerias com outras regiões, todos os docentes possuem vinculações informais com pesquisadores de outras regiões do Brasil, que também se concretizam em projetos, publicações, participação em eventos e bancas. Atualmente temos parcerias com: Universidade Federal de Rondônia (UNIR), Universidade Federal do Pará (UFPA), Universidade de Brasília (UnB), Universidade Federal da Bahia (UFBA), Universidade Federal de Pernambuco (UFPE), Universidade Federal do Ceará (UFC), Universidade Federal do Rio Grande do Sul (UFRGS), Universidade de São Paulo (USP) da capital paulista e de Ribeirão Preto, Universidade Estadual de Campinas (UNICAMP), Pontifícia Universidade Católica de Campinas (PUCCamp), Universidade Federal de São Carlos (UFSCar), Centro Latino-Americano de Estudos de Violência e Saúde Jorge Careli (ENSP-Fiocruz Claves), Universidade Federal Rural do Rio de Janeiro (UFRRJ). Assim, nota-se que ainda concentramos nossas parcerias com instituições do Sudeste, por terem elas mais força na pós-graduação.

Como estratégia para fortalecimento dos PPGs na região norte, aprovamos proposta no edital $n^{\circ} 21 / 2018$ Programa Nacional de Cooperação Acadêmica na Amazônia (PROCAD Amazônia), entre o PPGPSI-UFAM, MAPSI-UNIR e o PPG em Processo de Desenvolvimento Humano e Saúde (PPGPDS/UnB). A intenção dessa cooperação é de que se possa avançar na consolidação dos PPGs envolvidos, com elaboração de estratégias, pesquisas e parcerias conjuntas para responder adequadamente aos desafios atuais e futuros, ao produzir conhecimentos que ajudem a compreender as culturas dos jovens amazônidas.

Parte dessa vinculação interestadual acontece pela participação em GTs da Associação Nacional de Pesquisas e Pós-Graduação em Psicologia (ANPEPP), cujo PPGPSI também é filiado, e que propiciam a participação de nossos docentes em eventos em conjunto, pesquisas e publicações. Nossos docentes participam dos seguintes GTs: Cotidiano e Práticas Sociais; Cultura, Pensamento e Linguagem na Contemporaneidade; Fenomenologia, Saúde e Processos Psicológicos; Juventudes e Pesquisas Participativas; Psicodinâmica do Trabalho; Psicologia Comunitária.

Por fim, destacamos as parcerias internacionais consolidadas até o momento. A primeira é por meio de uma Cooperação Técnica (sem repasse de recursos financeiros) firmada entre a Universidad de La Cuenca del Plata (UCP) na Argentina e a UFAM, com vigência de cinco anos (2018-2023). Fruto dessa cooperação, se organizarão eventos internacionais, publicações conjuntas, trânsito entre docentes e discentes nas duas universidades e participação em reuniões, aulas e orientações utilizando recursos online. Está em vigência um projeto conjunto no âmbito da América Latina relacionando a Psicologia aos contextos rurais, que tem rendido aprendizagem mútua entre essas instituições (CAPES, 2018).

Outra parceria ainda não institucionalizada formalmente tem acontecido com os pesquisadores do Research Institute for Health and Social Change (RIHSC) da Manchester Metropolitan University (MMU) no Reino Unido, que vem se intensificando em ações desde 2014 após a realização de estágio pós-doutoral de uma das docentes. Atualmente, está em curso projeto com recursos da Fundação de Amparo à Pesquisa do Estado do Amazonas (FAPEAM), que visa a produção científica conjunta acerca de Arte e Intervenção em contextos de vulnerabilidade. Como desdobramentos destas ações de intercâmbio têm sido realizadas reuniões de pesquisa via videoconferência, co-orientações informais de projetos de mestrandos e organização de evento internacional, visitas internacionais nos dois países.

Para finalizar, tem havido intercâmbio informal também, por projetos em parceria e publicações, com as seguintes instituições: Universidad Nacional de Entre Ríos (UNER) na Argentina, Universidade de La Salle (México), Universidad de Havana (Cuba), Comunity University of New York (CUNY) nos Estados Unidos e Universidade do Algarve (em Faro, Portugal).

\section{Principais produções acadêmicas e inserção social}

No âmbito da divulgação de pesquisas acadêmicas, temos intensificado o esforço de publicações de artigos, livros e capítulos a partir do quadriênio 2017-2020, decorrente de avaliação ruim pelo relatório quadrienal de 2013-2016. Além de termos preenchido incorretamente o Portal Sucupira - antigamente Coleta Capes -, tivemos baixo nível de produtividade e baixa qualidade dos itens publicados. A partir de autoanálise do corpo docente e discente, diagnosticamos que nos faltou ousadia e coragem de, primeiramente, enviar textos e, em segundo, para revistas de bom qualis. De certa forma, a expectativa de um produtivismo exacerbado sem sentido nos imobilizou por um tempo. 
Aos poucos, temos buscado desenvolver estratégias para ampliarmos a qualidade e quantidade de nossas publicações. Entre estas estão: (a) incentivo à publicação com os discentes, por trabalhos de revisão crítica de literatura ou de resultados de pesquisa; (b) publicação por parcerias intra e interinstitucionais; (c) edição anual de livro com textos do PPGPSI e de outros convidados. Examinamos também que a escolha de qual veículo utilizar para envio dos textos depende da qualidade do material produzido.

Dessa feita, entre as cinco produções mais relevantes escolhidas nos relatórios de 2017 e 2018, temos como artigos de parcerias interinstitucionais os textos nacionais de Cardoso e Silva Filho (2018) e Freires, Silva Filho, Monteiro, Loureto e Gouveia (2017), enquanto texto internacional de Sampaio, Nina e Moraes (2017). Como produções com discentes em revistas nacionais temos Neves e Silva (2018) e, em revistas internacionais, temos Souza e Calegare (2018). Dos livros organizados e que selecionamos como representativos de temáticas regionais, fruto de parcerias interinstitucionais, temos as obras de Calegare e Albuquerque (2018), Gutierrez et al. (2017), Gutierrez e Ribeiro (2018) e Mascarenhas e Araújo (2017) e um capítulo em livro de bom qualis, o texto de Calegare (2017).

Quanto à inserção social, recebemos feedbacks da avaliação de 2013-2016 de que precisamos preencher melhor o Portal Sucupira para expressar todas as atividades que realizamos, pois temos muitas ações nesse item, porém mal descritos. Atualmente, nosso PPGPSI se destaca em atividades de inserção social envolvendo as seguintes ações de:

(a) Participação em Conselho Consultivo de duas Unidades de Conservação (UCs) federais, em Conselho Estadual de Segurança Alimentar e Nutricional, Comissão Interinstitucional de Educação Ambiental do Estado do Amazonas, Comitê Municipal de Enfrentamento à Mortalidade Relacionada ao Trabalho, Conselho Nacional de Defesa dos Direitos da Criança e do Adolescente, Conselho Federal de Psicologia; (b) Atuação junto às políticas públicas ligadas à Rede de Atenção Psicossocial no Estado do Amazonas, ao Centro de Referência em saúde do Trabalhador, à Secretaria Estadual de Justiça e Cidadania, ao Tribunal de Justiça do Estado do Amazonas, ao Centro de Atenção Psicossocial Infanto Juvenil (CAPSi) e o Álcool e Drogas (CAPS AD); (c) Cursos e palestras voltados ao público em geral em distintas instituições, como aquelas voltadas à saúde do trabalhador, à capacitação para o enfrentamento à violência sexual contra crianças e adolescentes, à educação para a promoção da equidade de gênero; (d) Projetos de intervenção junto a comunidades necessitadas, como por exemplo em grupo de apoio às crianças com câncer, em comunidade indígena na cidade de Manaus, em áreas de ocupação de habitação, em comunidade ribeirinha no interior do Amazonas e até na comunidade acadêmica da FAPSI - em projeto integrado por mestrandos, graduandos, servidores e professores da UFAM, para promover, saúde e bem-estar no ambiente acadêmico, pela integração de Arte e Psicologia; (e) Eventos de divulgação científica junto ao público em geral, como aqueles envolvendo discussões a respeito de raça-etnia, direitos humanos, arte e cultura; (f) Na atenção à educação básica, pela participação no Programa Nacional de Formação de Professores da Educação Básica.

Como se observa pela suscinta descrição, contida nos últimos relatórios submetidos ao Portal Sucupira para este quadriênio, o PPGPSI se destaca por inúmeras ações de inserção social. O que nos falta é elaborarmos melhores descrições do que fazemos. Uma dificuldade comum encontrada por nós, compartilhada por pessoas de outros PPGs, é que não temos um feedback personalizado de como e o que melhorar em nosso programa e nem dos relatórios anuais enviados. Uma solução tem sido chamar avaliadores externos, que com uma leitura cuidadosa de nossos relatórios podem nos ajudar com dicas para uma elaboração mais diretiva do relatório e pontos de melhorias de nossas ações, demonstrando o que fazemos no PPGPSI.

\section{Desafios e perspectivas}

A partir de autoavaliação docentes e de contribuições de avaliadores externos (Ufam, 2019), levantamos três grandes desafios para que o PPGPSI, um programa pós-graduação no localizado na região amazônica, incremente sua produção intelectual: (a) a produção de conhecimento socialmente relevante para a Amazônia; (b) a implementação de intercâmbio cientifico com pesquisadores e universidades de outros estados da região norte e de outras regiões do Brasil; (c) o desenvolvimento de planos estratégicos de internacionalização como meio de melhorar a qualidade do curso e de conferir maior visibilidade internacional à pesquisa científica realizada nesta região.

A formulação e implementação de políticas científicas e de formação de recursos humanos deve

Estudos de Psicologia, 24(2), abril a junho de 2019, 125-135 
considerar as especificidades de cada região. Em se tratando da região Norte e da Amazônia, destaca-se a diversidade de características de cada estado em termos de condições de deslocamento, de comunicação, de meio ambiente, de história de contato com os povos indígenas, de relação entre as etnias. De acordo com a Academia Brasileira de Ciências (2008), diante da importância da Amazônia e do reconhecimento de que o Brasil possui a maior biodiversidade do planeta, é fundamental que o país a conheça cientificamente: "isso supõe a presença de centros de pesquisa e o fortalecimento das estruturas universitárias na Amazônia como um todo" (p. 29).

Nós que vivemos na região Norte sabemos que há isolamento da Amazônia em relação ao restante do Brasil, construído em um longo e sangrento processo histórico de colonização que se estende até os dias atuais. Isso se reflete também no baixo fomento à pesquisa, em especial sobre os povos amazônicos, e no acesso restrito da população amazônica residente no interior à formação de nível superior (Silva, 2017). Assim, apesar do reconhecimento da relevância da Amazônia, verifica-se um baixo investimento em pesquisa no âmbito das Ciências Humanas e Sociais na região.

Abramovay (2018), ao falar do papel estratégico das populações tradicionais, afirma que a floresta e as populações amazônicas "representam não apenas utilidade econômica ou ecossistêmica, mas uma riqueza cultural que se exprime na diversidade das línguas, dos costumes e da própria cultura material dos povos da floresta" (p. 29). A preservação dos ensinamentos e da sabedoria dos povos amazônicos, portanto, deve ser pensada em conjunto com a proteção da floresta. Isso indica que um dos desafios para nosso PPGPSI é produzir conhecimentos pelo diálogo de saberes com os povos da floresta, que possuem uma relação intrínseca com seus territórios. Portanto, devemos seguir buscando investigar os fenômenos psicossociais sempre integrando o sujeito, o coletivo e o ambiente, atravessado pelas mais diversas dimensões de nossa existência: cultura, economia, política.

Ao analisar as oportunidades e as limitações da geração de conhecimento sobre as sociedades amazônicas, que são sociedades complexas, identifica-se que prevalecem as pesquisas sobre o meio ambiente em detrimento dos estudos sobre as pessoas. A criação da Fundação de Amparo à Pesquisa do Estado do Amazonas (FAPEAM), em 2002, foi uma importante medida para o incentivo à produção de conhecimento, entretanto ao longo da sua existência poucos editais contemplaram a área das ciências humanas.
De forma geral, os brasileiros de outras regiões conhecem pouco a região norte e a Amazônia. Muitas vezes, a região é apresentada de forma exótica e as pessoas são tratadas como objeto. Compreender as sociedades amazônicas exige epistemologias que considerem os compromissos ético-políticos da pesquisa científica e a importância de contribuir para o desenvolvimento sustentável. Descolonizar as epistemologias é um dos caminhos para avançarmos na valorização das identidades amazônicas.

Outro desafio refere-se à implementação de intercâmbio científico com pesquisadores e universidades de outros estados da região Norte e de outras regiões do Brasil. No contexto amazonense, constata-se: isolamento entre a capital e demais municípios do estado em razão do alto custo e o tempo de deslocamento entre os municípios, dificuldades de acesso à comunicação e uso da internet; a quase informalidade na organização do transporte fluvial. Esses fatores causam impactos na pesquisa que, em função do alto custo do deslocamento entre os municípios e a capital com passagens aéreas caras e deslocamentos demorados via fluvial, exige um investimento maior para sua viabilização. Tal cenário requer o entendimento das agências financiadoras de que parte considerável dos recursos financeiros dos programas e projetos são destinados aos largos e dispendiosos deslocamentos (Calegare, Higuchi, \& Forsberg, 2013). Situações como essas repercutem nas condições de funcionamento de um PPG no estado do Amazonas exigindo esforços para superação de obstáculos para a realização da pesquisa em regiões mais isoladas, como por exemplo, nas áreas indígenas e regiões ribeirinhas devido ao alto custo com transporte e estadia.

Na relação com PPGs de outras regiões, vivenciamos outros desafios. $O$ alto custo de passagens aéreas muitas vezes dificulta a participação em bancas de outros PPGs ou em eventos científicos. Isso tem desencorajado a realização de eventos nacionais em Manaus. Como já expressamos acima, considera-se que o estado do Amazonas e sua capital sejam isolados demais do restante do país, o que cria uma distância não apenas geográfica, mas psicossocial.

Buscando avançar no processo de internacionalização, o PPGPSI vem empreendendo esforços para a articulação entre pesquisadores/as que atuam nos países amazônicos, com a diversidade étnico-racial e suas implicações, a fim de dar visibilidade qualificada desta realidade em nível nacional e internacional. Nosso PPG atualmente é constituído por duas linhas de 
pesquisa e almeja a ampliação da pesquisa e da produção intelectual, a fim de melhorar seu desempenho na avaliação da CAPES. Para tanto, temos como meta estratégica criar um periódico científico na área da Psicologia, de gerência interinstitucional com as outras universidades de nossa região; e também credenciar o doutorado em Psicologia, pelo aumento da nota na avaliação da CAPES, com vistas ao desenvolvimento de nossa potencialidade acadêmica.

A partir da parceria estabelecida no programa de cooperação acadêmica, o Procad Amazônia, pretende-se enfrentar esse desafio e ampliar a formação de recursos humanos e a produção intelectual nos estados do Amazonas e Rondônia, que respondam aos desafios da Amazônia na perspectiva nacional e internacional. Os resultados dessa e de outras pesquisas em andamento poderão colaborar para uma oferta de educação de ensino superior identificado com as demandas da Amazônia, com a promoção de um desenvolvimento sustentável e que contemple a valorização dos conhecimentos e saberes das comunidades indígenas e tradicionais. Também se acredita que o fomento à permanência dos jovens egressos das universidades nos municípios e comunidades de origem, a valorização dos povos amazônicos e de seus conhecimentos sobre a floresta e as águas favorecerão a conservação dos recursos naturais, a atenção às necessidades das comunidades locais e o desenvolvimento em termos ambientais, culturais, políticos e econômicos (CAPES, 2018).

Com a finalidade de aprimorar a formação pós-graduada, temos planejado aulas conjuntas com uso de tecnologias digitais e analógicas, proporcionando estudo e discussões com a participação de professores-pesquisadores e estudantes de outras universidades nacionais e estrangeiras. Com esses esforços, estamos fortalecendo os passos na colaboração interinstitucional com outras universidades, por meio de parcerias entre os pesquisadores sêniores e estudantes, ou entre os estudantes em formação nas diferentes pós-graduações. Nossa meta é o aperfeiçoamento na produção de conhecimentos e o fomento à formação de futuros grupos de colaboração científica com outras universidades de países amazônicos e de outras regiões, possibilitando o incremento na avaliação do PPGPSI, o futuro credenciamento do doutorado em psicologia no Amazonas e a internacionalização da produção acadêmica.

\section{Referências}

Abramovay, R. (2018). A Amazônia precisa de uma economia do conhecimento da natureza. São Paulo: Alana, APIB, Artigo 19, Conectas Direitos Humanos, Engajamundo, Grennpeace, ISA, IEA, PPG em ciência ambiental da Universidade de São Paulo.

Academia Brasileira de Ciências. (2008). Amazônia: desafio brasileiro do Século XXI. São Paulo: Fundação Conrado Wessel.

Balbachevsky, E. (2005). A pós-graduação no Brasil: novos desafios para uma política bem-sucedida. In C. Brock \& S. Schwartzman (Orgs.), Os desafios da educação no Brasil (pp. 275-304). Rio de Janeiro: Nova Fronteira.

Bardin, L. (2011). Análise de conteúdo (ed. revisada e ampliada). São Paulo: Edições 70.

Calegare, M. G. A. (2017). Rumo a uma abordagem psicossocial da florestalidade (ruralidade) amazônica. In E. F. Rasera, M. S. Pereira, \& D. Galindo (Orgs.), Democracia participativa, estado e laicidade? Psicologia social e enfrentamentos em tempos de exceção (pp. 285-300). Porto Alegre: Abrapso.

Calegare, M. G. A., \& Albuquerque, R. (Orgs.). (2018). Processos psicossociais na Amazônia: reflexões sobre raça, etnia, saúde mental e educação. Embu das Artes, SP; Manaus: Alexia Cultural, EDUA.

Calegare, M. G. A., Higuchi, M. I. G., \& Forsberg, S. S. (2013). Desafios metodológicos ao estudo de comunidades ribeirinhas amazônicas. Psicologia \& Sociedade, 25(3), 571-580. doi: 10.1590/ S0102-71822013000300011

Calegare, M. G. A., \& Tamboril, M. I. B. (2017). Formação, atuação e produção do conhecimento em Psicologia Social na Amazônia brasileira: retalhos da nossa história. Textos e Debates, 31, 11-31. doi: 10.18227/2217-1448ted.v1i31.4254

Cardoso, L. M., \& Silva Filho, J. H. (2018). Satepsi e a qualidade técnica dos testes psicológicos no Brasil. Psicologia: Ciência e Profissão, 38(spe), 40-49. doi: 10.1590/1982-3703000209112

Coordenação de Aperfeiçoamento de Pessoal de Nível Superior CAPES. (2017). Plataforma Sucupira. Coleta de Informações 2017. Recuperado de https://sucupira.capes.gov.br/sucupira/public/ consultas/coleta/envioColeta/dadosFotoEnvioColeta.jsf

Coordenação de Aperfeiçoamento de Pessoal de Nível Superior CAPES. (2018). Plataforma Sucupira. Coleta de Informações 2018. Recuperado de https://sucupira.capes.gov.br/sucupira/public/ consultas/coleta/envioColeta/dadosFotoEnvioColeta.jsf

Freires, L. A., Silva Filho, J. H., Monteiro, R. P., Loureto, G. D. L., \& Gouveia, V. V. (2017). Ensino da avaliação psicológica no Norte brasileiro: analisando as ementas das disciplinas. Avaliação Psicológica, 16(2), 205-214. doi: 10.15689/AP.2017.1602.11

Freitas, M. F. Q., \& Souza, J. (2018). Pensar a formação e a pesquisa na pós-graduação stricto sensu. Educar em Revista, 34(71), 9-18. doi: 10.1590/0104-4060.62549

Gutierrez, D. M. D., Oliveira, F. R. M., Souza, E. O., Andrade, F.C., Carvalho, G. F., \& Leão, M. S. (2017). Tecnologias sociais do INPA para a Amazônia: uma visão diagnóstica. Manaus: INPA. Recuperado de http://portal.inpa.gov.br/arquivos/LIVRO-Tecnologias_Sociais_INPA_ Amazonia.pdf

Gutierrez, D. M. D., \& Ribeiro, J. H. S. (Orgs.). (2018). Suicídio: diálogos interdisciplinares. Manaus: Edua.

Mascarenhas, S. A. N., \& Araújo, J. N. (Orgs.). (2017). Desafios para o exercício da cidadania, qualidade de vida e inclusão socioeconômica na Amazônia. Pesquisa em educação, psicologia, sociedade, cultura e ambiente. São Paulo: Loyola. 
Ministério da Educação (2010). Coordenação de Aperfeiçoamento de Pessoal de Nível Superior-CAPES. Plano Nacionalde Pós-GraduaçãoPNPG 2011-2020. Brasília, DF: CAPES. Recuperado de https://www.capes.gov.br/plano-nacional-de-pos-graduacao

Ministério da Educação (2016). Coordenação de Aperfeiçoamento de Pessoal de Nível Superior - CAPES. Diretoria de Avaliação. Documento de Área - Psicologia. Recuperado de http://www.capes. gov.br/images/documentos/Documentos_de_area_2017/PSIC_ docarea_15fev2017.pdf

Neves, A. L. M., \& Silva, I. R. (2018). Projetos de igualdade de direitos às pessoas LGBT: significados das/os professoras/es sobre o seu protagonismo. Estudos \& Pesquisas em Psicologia, 18(2), 477-494. doi: 10.12957/epp.2018.38808

Sampaio, C. R. B., Nina, S. F. M., \& Moraes, R. D. (2017). Work relations and helping in the lives of Amazon rural women workers. Interpersona: An International Journal on Personal Relationships, 11(supp1), 11-21. doi: 10.5964/ijpr.v11isupp1.248
Silva, I. R. (2017). Psicologia Escolar: possibilidades de atuação profissional. Manaus: EDUA.

Souza, J. C., \& Calegare, M. G. A. (2018). Inserción social de los refugiados colombianos en Manaos, Brasil. Universitas Psychologica, 17(4), 1-10. doi: 10.11144/Javeriana.upsy17-4.isrc

Universidade Federal do Amazonas - UFAM. (2008). Pró-Reitoria de Pesquisa e Pós-Graduação. Faculdade de Educação. Departamento de Psicologia. Proposta do programa de pós-graduação stricto sensu em Psicologia - documentação complementar ao APCN/2008. Manaus: Autor.

Universidade Federal do Amazonas - UFAM. (2016). Programa de Pós-Graduação em Psicologia. Regimento Interno. Manaus: Autor. Recuperado de https://drive.google.com/file/d/1EkOaLSZEBliGyW eYslrlvK2VRkYlgaAo/view

Universidade Federal do Amazonas - UFAM. (2019). Programa de Pós-Graduação em Psicologia. Avaliação 10 anos do PPGPSI (relatório técnico). Manaus: Autor.

Gisele Cristina Resende, Doutora em Psicologia em Saúde e Desenvolvimento pela Universidade de São Paulo - USP, é Professora Adjunta na Faculdade de Psicologia da Universidade Federal do Amazonas - UFAM.

Endereço para correspondência: Av. Rodrigo Otávio, 6200 Setor Sul - Campus Universitário - Bloco X - FAPSI. Coroado. Manaus / AM CEP 69.080-900. Email: giseleresende@ufam.edu.br

Iolete Ribeiro da Silva, Doutora em Psicologia pela Universidade de Brasília - UnB, é Professora Associada na Faculdade de Psicologia da Universidade Federal do Amazonas - UFAM. Email: ioleteribeiro@ufam.edu.br

Marcelo Gustavo Aguilar Calegare, Doutor em Psicologia Social pela Universidade de São Paulo - USP, é Professor Adjunto da Faculdade de Psicologia da Universidade Federal do Amazonas - UFAM. Email:mgacalegare@ufam.edu.br

Recebido em 05.jun.19 Revisado em 19.jul.19 Aceito em 18.set.19 